\title{
Comparison of reduced-toxicity conditioning protocols using fludarabine, melphalan combined with thiotepa or carmustine in allogeneic hematopoietic cell transplantation
}

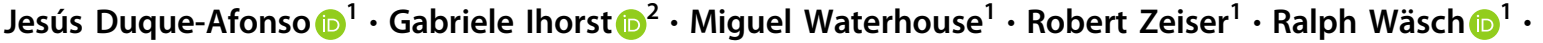 \\ Hartmut Bertz ${ }^{1} \cdot$ Mehtap Yücel $^{1} \cdot$ Thomas Köhler $^{3} \cdot$ Joachim Müller-Quernheim $^{3} \cdot$ Reinhard Marks $^{1} \cdot$ Jürgen Finke $^{1}$
}

Received: 8 May 2020 / Revised: 5 June 2020 / Accepted: 16 June 2020 / Published online: 26 June 2020

(c) The Author(s), under exclusive licence to Springer Nature Limited 2020

\begin{abstract}
The age of patients undergoing allogeneic hematopoietic cell transplantation (allo-HCT) has increased during the last decades, mainly due to improved reduced-intensity/toxicity conditioning protocols. A reduced-intensity conditioning based on fludarabin, carmustin/BCNU and melphalan (FBM) has been previously developed at our institution. Since we observed detrimental effects in individual patients with compromised lung function, efforts have been made in order to replace BCNU by thiotepa (FTM) to reduce toxicity. In this study, we retrospectively analyzed the outcome, GvHD incidence, lung function and organ toxicity of patients with a median age of 62 years (range 21-79) transplanted for malignant disease $(96.7 \%, 62.3 \%$ in intermediate/advanced disease stage) at our institution after conditioning with FBM $(n=136)$ or FTM $(n=105)$ between 2013 and 2017. Median follow-up was 868 days (range 0-2615). In multivariate analysis for overall survival, no difference was detected between both conditioning protocols in the presence of impaired lung function, age, lower performance, and liver disease previous allo-HCT. In the subgroup analysis, FTM was not inferior to FBM in patients with pulmonary disease prior allo-HCT, lymphoid malignancies, and higher comorbidity index. In conclusion, the reduced-intensity FBM and FTM conditioning protocols show adequate antineoplastic efficacy and are suitable for patients with impaired lung function.
\end{abstract}

\section{Introduction}

The age of patients undergoing hematopoietic cell transplantation has increased during the last decades, due to reduced-intensity conditioning protocols, which allow hematopoietic cell transplantation in older patients and with reduced performance status [1]. Current research is focused

Supplementary information The online version of this article (https:// doi.org/10.1038/s41409-020-0986-2) contains supplementary material, which is available to authorized users.

$\triangle$ Jesús Duque-Afonso

jesus.duque.afonso@uniklinik-freiburg.de

1 Department of Hematology/Oncology/Stem Cell Transplantation, Faculty of Medicine and Medical Center, University of Freiburg, Freiburg, Germany

2 Clinical Trials Unit, Faculty of Medicine and Medical Center, University of Freiburg, Freiburg, Germany

3 Department of Pneumology, Faculty of Medicine and Medical Center, University of Freiburg, Freiburg, Germany to develop conditioning protocols with reduced toxicity but with sufficient anti-leukemic effect [2].

BCNU (bis-chloroethylnitrosourea or carmustine) is an alkylating agent that has been successfully used in combination with alkylating agents in conditioning regimens for the treatment of lymphomas and multiple myeloma [3-5] and in the treatment of brain tumors [6]. BCNU is a lipophilic substance, which cross the blood-brain barrier and produces a prolonged and cumulative myelosuppression [7]. However, high dose of $\mathrm{BCNU}$ in conditioning regimen and cumulative dose of $\mathrm{BCNU}$ for the treatment of glioblastoma have been associated with severe pulmonary toxicity $[8,9]$.

Thiotepa ( $\mathrm{N}, \mathrm{N}^{\prime} \mathrm{N}^{\prime}$-triethylenethiophosphoramide) is also an alkylating agent with antineoplastic activity [10], immunosuppressive effects and ability to penetrate the blood-brain barrier [11]. Moreover, thiotepa has a dosedependent non-hematopoietic toxicity profile, with mucositis the most frequent complication in doses of $10-15 \mathrm{mg} /$ $\mathrm{kg}$ followed by sinusoidal obstructive syndrome, transient liver, renal and cardiovascular toxicities in higher doses [12-14]. Due to these properties, thiotepa has been incorporated as chemotherapy agent in conditioning protocols 
Table 1 Clinical characteristics of the patient cohort at the time of alloHCT.

\begin{tabular}{|c|c|c|c|}
\hline \multicolumn{4}{|l|}{ Clinical characteristics } \\
\hline Conditioning protocol & FBM & FTM & $p$ value \\
\hline Total patients & 136 & 105 & \\
\hline $\begin{array}{l}\text { Age at allo-HCT, median } \\
\text { (range) }\end{array}$ & $64(25-79)$ & $59(21-78)$ & 0.001 \\
\hline Age $<55$ years, $n(\%)$ & $23(16.9)$ & $40(38.9)$ & 0.001 \\
\hline Patient sex male, $n(\%)$ & $79(58.1)$ & $64(60.9)$ & 0.65 \\
\hline Donor sex male, $n(\%)$ & $79(58.1)$ & $65(61.9)$ & 0.55 \\
\hline $\begin{array}{l}\text { Donor recipient sex pair female to } \\
\text { male, } n(\%)\end{array}$ & $30(22.1)$ & $24(22.8)$ & 0.88 \\
\hline Primary disease, $n(\%)$ & & & 0.001 \\
\hline Myeloid & $121(89.0)$ & $68(64.8)$ & \\
\hline Lymphoid & $12(8.8)$ & $32(30.5)$ & \\
\hline Others & $3(2.2)$ & $5(4.8)$ & \\
\hline $\begin{array}{l}\text { Disease stage before allo-HCT, } \\
n(\%)\end{array}$ & & & 0.04 \\
\hline Early & $61(45.1)$ & $30(30.1)$ & \\
\hline Intermediate & $15(11.5)$ & $15(17.8)$ & \\
\hline Late & $60(43.4)$ & $60(52.0)$ & \\
\hline Prior therapy lines, $n(\%)$ & & & 0.001 \\
\hline Untreated & $34(25.0)$ & $14(13.3)$ & \\
\hline 1st line & $60(44.1)$ & $39(37.1)$ & \\
\hline 2nd line & $20(14.7)$ & $10(9.5)$ & \\
\hline$\geq 3$ rd line & $22(16.1)$ & $41(39.0)$ & \\
\hline $\begin{array}{l}\text { Prior therapy cycles, mean } \\
\text { (range) }\end{array}$ & $3.0(0-21)$ & $4.5(0-20)$ & 0.004 \\
\hline $\begin{array}{l}\text { Prior therapy } \geq 3 \text { cycles, } \\
n(\%)\end{array}$ & $33(22.1)$ & $42(40.0)$ & 0.01 \\
\hline Prior auto-HCT & $4(2.9)$ & $21(20.0)$ & 0.001 \\
\hline $\begin{array}{l}\text { Follow-up in days, median } \\
\text { (range) }\end{array}$ & $955(5-2615)$ & $762(0-1961)$ & 0.001 \\
\hline Donor, $n(\%)$ & & & 0.02 \\
\hline Related & 37 (24.6) & $19(15.3)$ & \\
\hline Unrelated & $99(75.4)$ & $80(76.7)$ & \\
\hline Haplo & 0 & $5(6.8)$ & \\
\hline Twin & 0 & $1(1.3)$ & \\
\hline $\begin{array}{l}\text { HLA-A, -B, -DR1, DQ1, } \\
n(\%)\end{array}$ & & & 0.05 \\
\hline Ident & $105(77.0)$ & $76(71.2)$ & \\
\hline Different & $31(22.9)$ & $23(20.5)$ & \\
\hline Haplo-ident & 0 & $5(6.8)$ & \\
\hline Twin & 0 & $1(1.3)$ & \\
\hline HLA-C, $n(\%)$ & & & 0.12 \\
\hline Ident & $116(95.1)$ & $68(93.2)$ & \\
\hline Different & $6(4.9)$ & $3(2.7)$ & \\
\hline Haplo-ident & 0 & $3(4.1)$ & \\
\hline EBMT disease risk score, $n(\%)$ & & & 0.20 \\
\hline $0-2$ & $15(11.5)$ & $6(6.8)$ & \\
\hline $3-4$ & $55(40.2)$ & $38(31.5)$ & \\
\hline $5-7$ & $66(48.4)$ & $61(61.6)$ & \\
\hline $\begin{array}{l}\text { GvHD prophylaxis with in vivo } \\
\text { T-cell depletion, } n(\%)\end{array}$ & $118(86.8)$ & $69(65.7)$ & 0.001 \\
\hline ATG & $99(72.8)$ & $68(64.7)$ & \\
\hline Alemtuzumab (campath) & 19 (13.9) & $1(0.9)$ & \\
\hline Methotrexate-based, $n(\%)$ & $30(22.1)$ & $1(0.9)$ & 0.001 \\
\hline $\begin{array}{l}\text { Karnofsky prior allo-HCT, } \\
n(\%)\end{array}$ & & & \\
\hline$\leq 90$ & $113(83.1)$ & $90(85.7)$ & 0.58 \\
\hline
\end{tabular}

Table 1 (continued)

\begin{tabular}{lccc}
\hline Clinical characteristics & & & \\
\hline Conditioning protocol & FBM & FTM & $p$ value \\
\hline$\leq 80$ & $45(33.1)$ & $47(44.7)$ & 0.07 \\
HCT-CI score, median (range) & $\mathbf{3 ( 0 - 1 0 )}$ & $\mathbf{4 ( 0 - 1 0 )}$ & $\mathbf{0 . 0 3}$ \\
HCT-CI score, $n(\%)$ & & & 0.40 \\
0 & $12(24.3)$ & $6(7.6)$ & \\
1 & $12(5.1)$ & $8(7.6)$ & \\
2 & $23(39.0)$ & $13(18.1)$ & \\
3 & $26(13.2)$ & $16(24.8)$ & \\
$\geq 4$ & $63(18.4)$ & $62(41.9)$ &
\end{tabular}

Statistical analysis of categorical variables was performed by Pearson's chi-square and Fisher's exact test; continuous variables by Student's T-test assuming a normal distribution.

allo-HCT allogeneic hematopoietic cell transplantation, $G v H D$ Graftversus-Host-Disease, $A T G$ anti-thymocyte anti-globulin, $H C T-C I$ hematopoietic cell transplantation comorbidity index, FBM Fludarabine, BCNU, Melphalan, FTM Fludarabine, Thiotepa, Melphalan.

prior autologous hematopoietic cell transplantation (autoHCT) $[15,16]$ and allogeneic hematopoietic cell transplantation (allo-HCT) [17, 18].

A reduced-intensity conditioning protocol based on fludarabine, BCNU, and melphalan (FBM) has been developed at our institution $[19,20]$. Although this protocol has been shown to have anti-leukemic activity [21] and adequate quality of life [22, 23], patients still developed post-transplant complications as lung function impairment [24, 25]. Recent efforts have been made in order to replace $\mathrm{BCNU}$ by thiotepa in conditioning protocols prior auto-HCT [26, 27]. Therefore, to reduce lung toxicity while maintaining anti-leukemic activity, a conditioning protocol for allo-HCT replacing the alkylating agent BCNU by thiotepa (FTM protocol) has been developed [28-30].

In this study, we analyzed and retrospectively compared the outcome of patients, cause of deaths, impact on GvHD incidence, organ function toxicity, hematopoietic and immune system reconstitution after conditioning with the reduced-intensity/toxicity protocols FBM and FTM at our institution during the same time period. Patients treated with the FTM protocol showed similar outcomes compared to FBM protocol, and allowed allo-HCT in patients with impaired lung function and/or lowperformance status.

\section{Material and methods}

\section{Patient selection and characteristics}

Patients included in the study were transplanted at the University of Freiburg Medical Center between January 1st 
Table 2 Pulmonary characteristics of the patient cohort at the time of allo-HCT.

\begin{tabular}{|c|c|c|c|}
\hline \multicolumn{4}{|l|}{ Pulmonary clinical characteristics } \\
\hline Conditioning protocol & FBM & FTM & $p$ value \\
\hline $\begin{array}{l}\text { Pulmonary disease prior allo- } \\
\text { HCT, } n(\%)\end{array}$ & $44(33.1)$ & 49 (46.7) & 0.04 \\
\hline Bact. pneumonia & $16(11.8)$ & $15(14.3)$ & \\
\hline Fungal pneumonia & $8(5.9)$ & $8(7.6)$ & \\
\hline Atypical pneumonia & $5(3.7)$ & $4(3.8)$ & \\
\hline PjP pneumonia & 0 & $2(1.9)$ & \\
\hline Influenza A pneumonia & 0 & $2(1.9)$ & \\
\hline Asthma/COPD & $1(0.7)$ & $13(12.3)$ & \\
\hline Pulmonary embolism & $5(3.7)$ & $6(5.7)$ & \\
\hline Pleural effusions & $4(2.9)$ & $3(2.8)$ & \\
\hline Previous tuberculosis & $4(2.9)$ & 0 & \\
\hline Bronchitis & $1(0.7)$ & $2(1.9)$ & \\
\hline Sleep apnea & $1(0.7)$ & $2(1.9)$ & \\
\hline Others & $4(2.9)$ & $5(4.8)$ & \\
\hline $\begin{array}{l}\text { Current and previous } \\
\text { smokers, } n(\%)\end{array}$ & $45(33.1)$ & 49 (46.7) & 0.04 \\
\hline $\begin{array}{l}\text { Pulmonary lung function test } \\
\text { before allo-HCT, } n(\%)^{\mathrm{a}}\end{array}$ & $120(89.5 \%)$ & $96(91.4 \%)$ & \\
\hline FEV1 (\% of predicted) & $97(53-145)$ & $95(23-138)$ & 0.02 \\
\hline FEV1/VCmax ratio & $0.75(0.49-1.03)$ & $0.74(0.18-0.99)$ & 0.05 \\
\hline FEV1/FVC ratio & $0.79(0.55-0.99)$ & $0.77(0.35-1.00)$ & 0.02 \\
\hline FVC (\% of predicted) & $103(47-159)$ & $99(43-147)$ & 0.02 \\
\hline MEF50 (\% of predicted) & $68.3(19-150)$ & $65.5(2.5-137)$ & 0.03 \\
\hline MEF25 (\% of predicted) & $44.8(9-137)$ & $45.1(9-103)$ & 0.38 \\
\hline DLCOcSB ( $\%$ of predicted) & $76.9(36-127)$ & $68.0(21-106)$ & 0.001 \\
\hline $\mathrm{RV}$ (\% of predicted) & $108.5(44-349)$ & $110(59-370)$ & 0.27 \\
\hline $\mathrm{RV} / \mathrm{TLC}$ ratio & $0.39(0.10-1.40)$ & $0.40(0.22-1.35)$ & 0.15 \\
\hline TLC ( $\%$ of predicted) & $99.9(60-159)$ & $100.0(71.5-159)$ & 0.53 \\
\hline Arterial $\mathrm{CO}_{2}, \mathrm{~mm} \mathrm{Hg}$ & $35(26-43)$ & $35(24-44)$ & 0.37 \\
\hline Arterial $\mathrm{O}_{2}, \mathrm{mmHg}$ & $77.7(62-106)$ & $79.0(59-102)$ & 0.20 \\
\hline
\end{tabular}

Statistical analysis of categorical variables was performed by Pearson's chi-square and Fisher's exact test; continuous variables by Student's T-test assuming a normal distribution.

allo- $H C T$ allogeneic hematopoietic cell transplantation, $R V$ residual volume, TLC total lung capacity, VCmax maximal vital capacity, $F E V 1$ forced expiratory volume in $1 \mathrm{~s}, F E V 1 / F V C$ FEV1/forced vital capacity (FVC), MEF50 mid-expiratory flow 50\%, MEF25 midexpiratory flow $25 \%, a \mathrm{CO}_{2}$ arterial carbon dioxide, $a \mathrm{O}_{2}$ arterial oxygen, $D L C O C S B$ diffusion capacity of carbon monoxide adjusted for hemoglobin level, FBM fludarabine, BCNU/carmustine, melphalan, FTM fludarabine, thiotepa, melphalan.

${ }^{a}$ Data are presented as median(range).

2013 and December 31st 2017 using reduced-intensity/ toxicity protocols FBM or FTM. We included all patients at first allo-HCT. All clinical data were prospectively collected and retrospectively analysed. Patient characteristics are described in Table 1 and Supplementary Table 1 and pulmonary clinical characteristics in Table 2. HCT-CI Score [31] and EBMT disease risk score [32] were evaluated as previously described by standard international guidelines. Causes of death were adapted from previously described criteria [33]. This study was conducted in accordance to the Declaration of Helsinki. Written consent was obtained from the subjects for data use in clinical research. All patients gave informed consent and the institutional review board of the University of Freiburg Medical Center approved reduced-intensity conditioning protocols. All data were evaluated as of March 31th 2020.

\section{Conditioning protocol, GvHD prophylaxis and Graft source}

Patients were stratified by attending physicians to receive conditioning according to FBM protocol (fludarabine $4 \times$ $30 \mathrm{mg} / \mathrm{m}^{2}, \mathrm{BCNU} /$ carmustine $2 \times 150 \mathrm{mg} / \mathrm{m}^{2}$ and melphalan by patients $>55$ years: $1 \times 110 \mathrm{mg} / \mathrm{m}^{2}$ or $<55$ years: $1 \times$ $140 \mathrm{mg} / \mathrm{m}^{2}$ ) or FTM (fludarabine $4 \times 30 \mathrm{mg} / \mathrm{m}^{2}$, thiotepa $2 \times 5 \mathrm{mg} / \mathrm{kg}$ and melphalan for patients $>55$ years: $1 \times$ $110 \mathrm{mg} / \mathrm{m}^{2}$ or $<55$ years: $1 \times 140 \mathrm{mg} / \mathrm{m}^{2}$ ). Caring physicians allocated patients to the two protocols depending mainly on previous therapies, pulmonary toxicity, and comorbidities. GCSF mobilized peripheral blood stem cell (PBSC) grafts were used in most of the cases $(n=239,99.2 \%)$ together with cyclosporine based GvHD prophylaxis (cyclosporine $5 \mathrm{mg} / \mathrm{kg}$ b.w. per day, starting day -3 , targeted through serum level between $250-350 \mathrm{ng} / \mathrm{ml}$ ) combined with methotrexate, mycophenolate mofetil, anti T-lymphocyte globulin (previously ATG-F, now ATLG, Grafalon) $[34,35]$ or alemtuzumab [36]. Two patients received an everolimus-based GvHD prophylaxis and two further patients a tacrolimus-based GvHD prophylaxis.

\section{Acute and chronic GvHD and organ toxicity}

Time to acute GvHD (aGvHD) and chronic GvHD (cGvHD) were defined as the number of days from transplantation to the occurrence of a GvHD episode with characteristic clinical aGvHD or cGvHD symptoms $[37,38]$. The grade of GvHD was classified according to Glucksberg's criteria [39]. Conditioning regimen-related toxicity by organ system within the first 30 days after alloHCT was assessed as previously described [40].

\section{Hematopoietic reconstitution, bone marrow chimerism, and regeneration of lymphocyte subpopulations}

Bone marrow chimerism was measured using standard PCR for microsatellite amplification as previously described [41]. Mixed chimerism was considered when recipient DNA was detected in $>1 \%$ of PCR reaction. Determination of leucocyte subsets was performed as previously described [42]. Gating of lymphocytes and estimation of the gated lymphocytes were performed according to international guidelines [43]. 


\section{Pulmonary function tests (PFTs)}

Pulmonary function tests (PFTs) including diffusion capacity for carbon monoxide were routinely performed according to international standards $[44,45]$ a week before allo-HCT and after 30 days, 3, 6, and 12 months, then repeated on the basis of clinical suspicion of pulmonary disease. PFT parameters were evaluated and expressed as percentage of predicted normal values, calculated using published equations [46], which were based on a population (caucasian, Middle Europe) very similar to the patients included in this study.

\section{Study Endpoints, Definitions, and Statistical Analysis}

Statistical analysis of patient characteristics, PFTs, cumulative incidences, and hazard ratios was conducted using STATA v11.0 (College Station, Texas, USA). Overall survival (OS) was defined as time from allo-HCT until death from any cause, and progression-free survival (PFS) was calculated as time from allo-HCT to death from any cause or relapse, whichever occurred first. For those patients not experiencing the event in question for OS or PFS during follow-up, time to last contact was used as a censored observation. Relapse was defined as detection of disease via cytological and histological assessment after allo-HCT; death without prior relapse was considered as a competing risk and denoted as non-relapse mortality (NRM). For aGvHD and cGvHD cumulative incidences, death without aGvHD/cGvHD was considered as competing risk.

The Cox proportional hazards regression model was used to estimate hazard ratios (HR) and confidence intervals (CI) for OS and PFS. We applied the Fine and Gray model to compare cumulative incidence rates in the presence of competing risks [47, 48] and presented subdistribution hazard ratios (SHR) for relapse incidence, NRM, aGvHD and $\mathrm{cGvHD}$ incidence.

Multivariate analyses for OS were conducted using Cox proportional hazards regression model using a backward selection strategy. Covariates including clinical characteristics and pulmonary function tests values with a univariate $p$ value $<0.1$ were included. HR and two-sided $95 \%$ confidence intervals of prognostic factors for OS were estimated.

Pearson's chi-square and Fisher's exact tests were used to compare categorical variables as appropriate [49] and Student's $T$-test to compare continuous variables assuming a normal distribution. Differences in PFTs before allo-HCT and at day +365 were analyzed using mixed-model for longitudinal analysis based on the maximum likelihood method.

\section{Results}

\section{Clinical features of patients by reduced toxicity conditioning protocols prior allo-HCT}

Between January 1st 2013 to December 31st 2017, 241 patients were conditioned with the reduced-intensity/toxicity protocols FBM $(n=136)$ or FTM $(n=105)$ at the Medical Center of the University of Freiburg (Germany). During the observation period, continuously increasing number of patients were treated with FTM. Therefore, patients treated with FBM had a longer follow-up compared to FTM-treated patients (median 955 vs. 762 days, $p<$ 0.001). The clinical features of the patients are described in Table 1 and Supplementary Table 1. Compared to patients conditioned with FBM, FTM-treated patients were younger and received less frequently GvHD prophylaxis with in vivo T-cell depletion, including alemtuzumab. Patients treated with FTM suffered more frequently lymphoid malignancies, had a late disease stage defined by EBMT score, received more chemotherapy cycles and had a higher HCT-CI score (Table 1). Patients with pulmonary diseases prior allo-HCT, current/previous smokers, and with impaired lung function were more frequently allocated in the FTM group, due to the patient selection by attending physicians to the presumably less pulmonary toxic protocol (Table 2).

\section{Impact of reduced toxicity conditioning protocol on outcome after allo-HCT}

In the univariate analysis, no differences could be demonstrated for OS between FTM and FBM-treated patients, despite of higher HCT-CI score and impaired lung function (Fig. 1a). To elucidate the impact of reduced-intensity/ toxicity conditioning protocols on OS and NRM considering other relevant clinical factors, we performed univariate and multivariate analyses by Cox proportional hazard regression (Supplementary Tables 2-4 and Table 3). Multivariate analysis adjusted for clinical and pulmonary factors impacting the overall survival (FEV $1<70 \%$ of predicted, DLCOcSB $<60 \%$ of predicted, HCT-CI liver, Karnofsky index $\leq 80 \%$ and age) showed no significant difference between both conditioning protocols on overall survival (Table 3, Fig. 1b). Most of the clinical characteristics affecting OS in the survival analysis also increase NRM (Supplementary Tables 3 and 4). Altogether, these data suggest that FTM conditioning is, at least, not inferior regarding outcome to FBM conditioning adjusted for adverse clinical factors.

To define which groups of patients are more suitable for conditioning with FTM compared to FBM, we performed subgroup analyses. Interestingly, FTM conditioning was not inferior in patients with impaired lung function as defined 
a

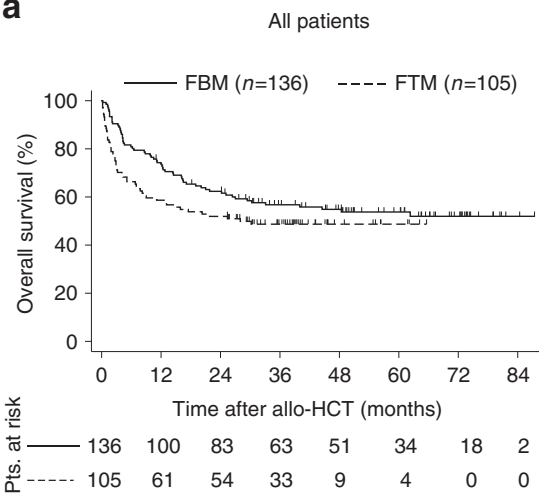

Fig. 1 Impact of reduced-intensity/toxicity conditioning on outcome. Kaplan-Meier curves represent overall survival for (a) unadjusted and (b) adjusted for parameters influencing mortality in multivariate analysis (FEV $1<70 \%$ of predicted, DLCOcSB $<60 \%$ of predicted, Karnofsky index $<80 \%$, HCT-CI liver, age $<55$ years) by

Table 3 Multivariate analysis for overall survival of clinical and lung function parameters.

\begin{tabular}{lrlll}
\hline & \multicolumn{4}{c}{ Hazard ratios for overall survival } \\
\cline { 2 - 5 } & $n$ & HR & $95 \%$ CI & $p$ value \\
\hline FEV1 < 70\% of predicted & 18 & 2.47 & $1.20,5.06$ & 0.02 \\
DLCOc SB $<60 \%$ of predicted & 35 & 1.87 & $1.15,3.03$ & 0.02 \\
Karnofsky index $\leq 80 \%$ & 92 & 1.70 & $1.11,2.58$ & 0.02 \\
HCT-CI score liver & 51 & 1.69 & $1.04,2.74$ & 0.03 \\
Age $<55$ years & 63 & 0.51 & $0.30,0.88$ & 0.02 \\
Conditioning with FTM & 105 & 1.31 & $0.85,2.58$ & 0.21
\end{tabular}

Clinical factors (Supplementary Table 2) with a $p$ value $<0.1$ in univariate analysis were included in the multivariate analysis with conditioning protocol as hypothesis covariate. Multivariate analysis following backward selection was performed using the Cox proportional hazards regression model.

HCT-CI hematopoietic cell transplantation comorbidity index, $D L C O C S B$ diffusion capacity of carbon monoxide adjusted for hemoglobin level, $F E V 1$ forced expiratory volume in $1 \mathrm{~s}, H R$ hazard ratios, $C I$ confidence intervals, FTM fludarabine, thiotepa, melphalan.

by HCT-CI lung, with pulmonary diseases prior allo-HCT, lymphoid malignances or with HCT-CI score of $\geq 4$ (Supplementary Figs. 1-4). These data indicate, that patients with initial impaired lung function and/or several comorbidities might profit by FTM conditioning.

\section{Cause of death by conditioning regimens}

Overall, 116 patients $(48.1 \%)$ died: 62 patients $(45.6 \%)$ in the FBM group and 54 patients $(51.4 \%)$ in the FTM group (Table 4). Mortality before day 100 after allo-HCT were mostly due to infections including pneumonia, sepsis, and fungal infections whereas mortality after day 100 were mostly due to relapses. No significant differences in cause

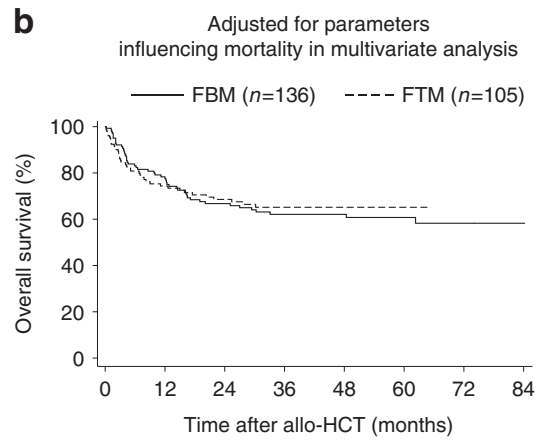

conditioning in all patients included in this study. Statistical analysis was performed for overall survival by log-rank test. Allo-HCT allogeneic hematopoietic cell transplantation, FBM fludarabine, BCNU, melphalan; FTM: fludarabine, thiotepa, melphalan; Pts., patients.

Table 4 Cause of mortality by conditioning regimen.

\begin{tabular}{ll}
\hline FBM $(n=62 / 136,45.6 \%)$ & FTM $(n=54 / 105,51.4 \%)$ \\
\hline $\begin{array}{l}\text { Relapse or progress } \\
(n=28,20.6 \%)\end{array}$ & Relapse or progress $(n=15,14.3 \%)$ \\
$\begin{array}{l}\text { Non-relapse mortality } \\
(n=34,25.0 \%)\end{array}$ & Non-relapse mortality $(n=39,37.1 \%)$ \\
$\begin{array}{l}\text { (a) Non-pulmonary } \\
(n=15,11.0 \%)\end{array}$ & (a) Non pulmonary $(n=20,19.0 \%)$ \\
GvHD $(6 \times)$ & CNS Bleeding complications $(2 \times)$ \\
Infection/sepsis $(3 \times)$ & CNS failure/Encephalopathy \\
CNS failure & GvHD $(7 \times)$ \\
Cardiac failure & Sepsis $(4 \times)$ \\
Peritonitis & Cardiac failure \\
Acute renal failure & CMV colitis \\
Unknown & Fungal infection $(2 \times)$ \\
Sudden death & Post-transplant lymphoproliferative \\
& disease \\
Unknown \\
(b) Pulmonary ( $n=19,14.0 \%)$ & $(b)$ Pulmonary $(n=19,18.1 \%)$ \\
Pneumonia/pneumonic & Pneumonia/pneumogenic \\
sepsis $(10 \times)$ & sepsis $(8 \times)$ \\
Interstial pneumonitis & Fungal pneumonia \\
Fungal pneumonia $(3 \times)$ & PjP-pneumonia \\
ARDS & ARDS $(4 \times)$ \\
Pulmonary embolism & Pulmonary embolism \\
Bronchial carcinoma & Bronchiolitis obliterans $(2 \times)$ \\
Bronchiolitis obliterans & Pulmonary failure \\
CMV pneumonia & Pneumothorax \\
\hline &
\end{tabular}

Table depicts cause of mortality by conditioning classified in relapse and non-relapse mortality including pulmonary and non-pulmonary causes. GvHD Graft-versus-Host-Disease, ARDS acute respiratory distress syndrome, $P j P$ pneumocystis jirovicii pneumonia, $C M V$ cytomegalovirus, $C N S$ central nervous system, $F B M$ fludarabine, BCNU/carmustine, melphalan, FTM fludarabine, thiotepa, melphalan. 


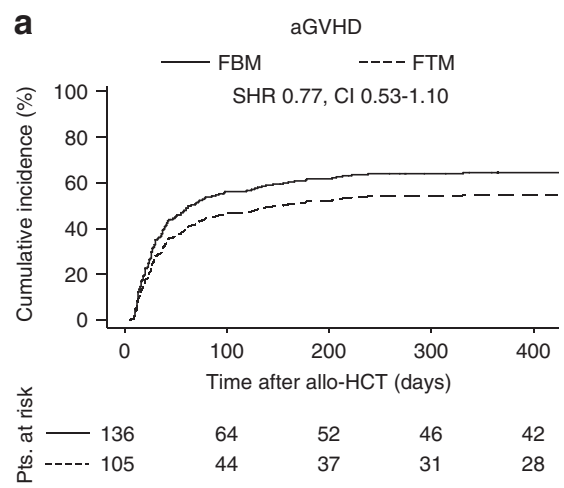

Fig. 2 Incidence of acute and chronic GvHD is not affected by conditioning FTM protocol. Cumulative incidences of (a) acute GvHD (aGvHD) and (b) chronic GvHD (cGvHD) were calculated for FBM and FTM conditioning protocols. Clinical evidence of aGvHD or cGvHD was computed as event, death was considered as competing

of death could be found between FBM and FTM patients, although the patients had different follow-up times.

\section{Impact of conditioning on GvHD, hematopoietic and immune system reconstitution}

Besides its hematopoietic stem cell toxicity, it has been postulated that thiotepa has immunsuppressive properties as conditioning chemotherapy $[50,51]$. Therefore, we assessed the impact of reduced toxicity conditioning protocols FBM and FTM on GvHD incidence, hematopoietic, and immune system reconstitution.

No significant differences were found in the cumulative incidence of aGvHD and cGvHD and severity and organs affected by aGvHD and cGvHD (Fig. 2 and data not shown) as well as in blood cells recovery and lymphocyte subpopulations (Supplementary Table 5) between both protocols in our patient cohort. In conclusion, the FTM conditioning protocol does not affect the incidence and organs affected by $\mathrm{aGvHD}$ and $\mathrm{cGvHD}$, the hematopoietic and immune system reconstitution compared to the FBM protocol.

\section{Impact of conditioning on lung function}

The main goal of using the FTM protocol at our institution was to reduce the risk of lung toxicity possibly caused by BCNU while maintaining anti-leukemic activity. Therefore, we investigated the impact of FTM- and FBM-conditioning on lung function in patients with available pulmonary function tests prior allo-HCT and 1 year after allo-HCT (Fig. 3, Supplementary Tables 6 and 7). As expected, due to selection of patients with impaired pre-transplant lung function for the FTM protocol, FEV1, FEV1/VCmax ratio, MEF50, and DLCOcSB were lower in the FTM-group. We hypothesize that causes for reduced lung function in FTM-

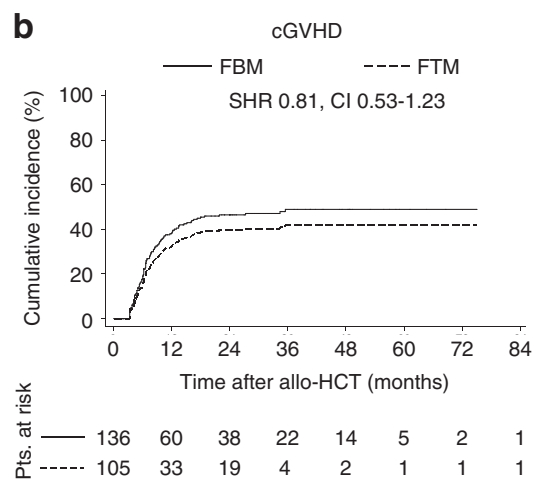

risk and time to last contact as censored event. Statistical differences were calculated by Fine and Gray regression model in the presence of competing-risks. SHRs represent the risk of FTM as compared to FBM. Pts. patients. FBM: fludarabine, BCNU/carmustine, melphalan; FTM fludarabine, thiotepa, melphalan.

treated patients prior allo-HCT were due to pulmonary diseases, smoking, use of lung toxic chemotherapies, irradiation, and previous auto-HCT among others. At 1 year after allo-HCT, statistically differences were still observed in obstructive parameters FEV1, FEV1/VCmax and in DLCOcSB (Fig. 3, Supplementary Table 6). Hence, dynamic changes of VCmax, FEV1, FVC, MEF50 in FBMtreated patients but not in FTM-treated patients were found to be statistically significant (Supplementary Table 7). Loss of $5.5 \%$ vital capacity in FBM-treated and $4.5 \%$ in FTM treated patients are on the order of the minimum clinically important difference (MCID) in terms of mortality for restrictive lung diseases [52].

In summary, patients treated with FTM protocol had impaired lung function compared to FBM treated patients prior allo-HCT. Significant decreases in several lung function parameters were observed in FBM-treated but not in FTM-treated patients at one year after allo-HCT.

\section{Regimen-related toxic effects by conditioning protocol}

Early toxicity within the first 30 days according to Bearman's regimen-related criteria [40] (Table 5) and frequency of complications after allo-HCT were assessed (Supplementary Table 8). Overall, toxicity related to liver and other organs including bladder, heart, lungs were similar in both groups. Patients conditioned with FTM-protocol developed more frequently mild and moderate mucositis compared to FBM-treated patients $(39.7 \%$ vs $63.8 \%, p<0.001$, Table 5). Regarding complications after allo-HCT, patients conditioned with FTM have less infections by HHV6-virus $(13.2 \%$ vs $3.8 \%, p<0.02)$ and more infections by HSV ( $4.4 \%$ vs $12.4 \%, p<0.03$ ). As expected due to worse performance status and concomitant comorbidities prior allo- 

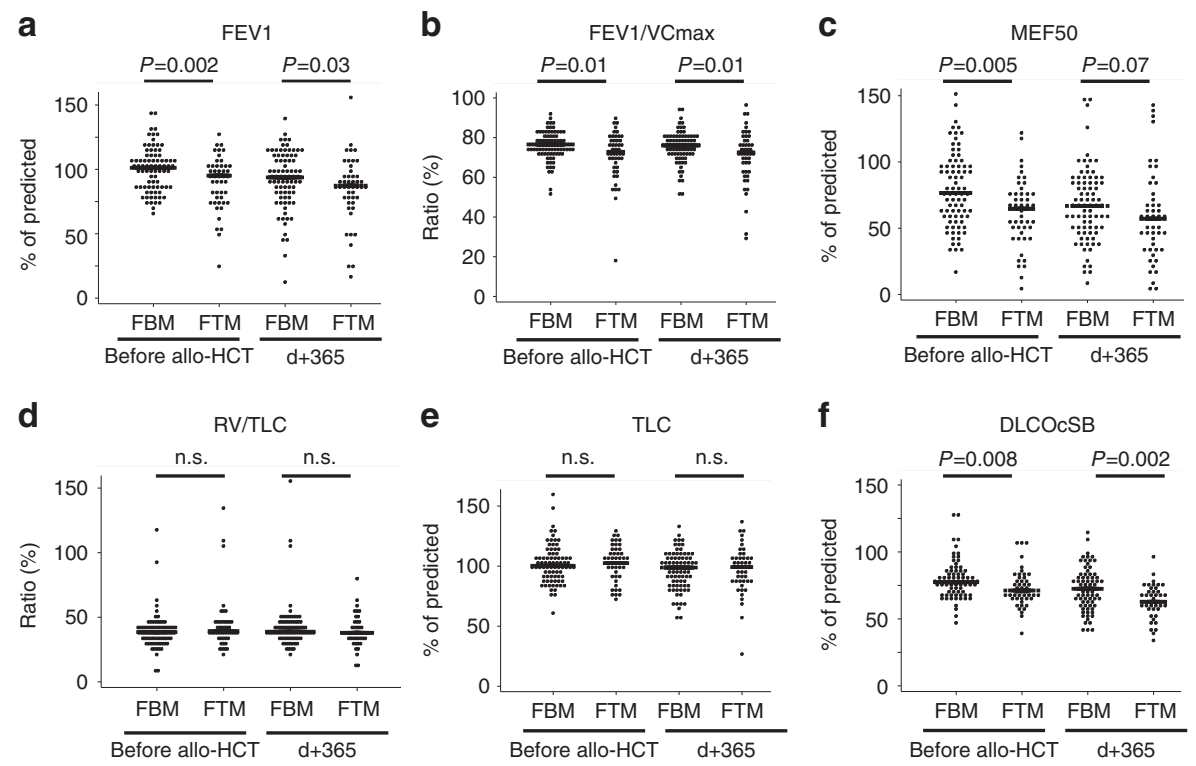

Fig. 3 Impact of conditioning protocol on lung function after alloHCT. Dot plots represent pulmonary function tests (PFT) values from patients before allo-HCT and at 1 year after allo-HCT. Only patients with lung function available at both time points were included in the analysis. PFT parameters were compared by conditioning protocol from patients with available PFT at both time points (FBM, $n=80 / 99$ alive at $\mathrm{d}+365,80.8 \%$; FTM, $n=47 / 61$ alive at $\mathrm{d}+365,77.0 \%)$. Each dot represent the indicated PFT value from a patient, horizontal bar represents the median. Statistical analysis were performed by Student's
$T$-test assuming a normal distribution. Allo-HCT allogeneic hematopoietic cell transplantation, FEV1 forced expiratory volume in $1 \mathrm{~s}$, FEV1/maximal vital capacity (), FEV1/forced vital capacity (FVC); MEF50, mid-expiratory flow 50\%; RV/TLC, residual volume (RV)/ total lung capacity (TLC); TLC, total lung capacity; DLCOcSB, diffusion capacity of carbon monoxide adjusted for hemoglobin level. FBM: fludarabine, BCNU/carmustine, melphalan; FTM: fludarabine, thiotepa, melphalan.

Table 5 Conditioning regimen related early toxicity by organ system within the first 30 days after allo-HCT.

\begin{tabular}{|c|c|c|c|c|c|c|c|c|c|}
\hline \multirow[b]{2}{*}{ Organ } & \multicolumn{4}{|c|}{$\operatorname{FBM}(n=136)$} & \multicolumn{4}{|c|}{ FTM $(n=105)$} & \multirow[b]{2}{*}{$p$ value } \\
\hline & Grade 1 & Grade 2 & Grade $3 / 4$ & Total & Grade 1 & Grade 2 & Grade 3/4 & Total & \\
\hline $\operatorname{Mucosa} N(\%)$ & $33(24.3)$ & $18(13.2)$ & $3(2.2)$ & $54(39.7)$ & $47(44.8)$ & $15(14.3)$ & $5(4.8)$ & $67(63.8)$ & 0.001 \\
\hline Kidney $N(\%)$ & $49(36.1)$ & $21(15.4)$ & $11(8.1)$ & $81(59.5)$ & $37(35.2)$ & $16(15.2)$ & $21(20.0)$ & $74(70.5)$ & 0.08 \\
\hline Liver $N(\%)$ & 39 (28.6) & $23(16.9)$ & $4(2.9)$ & $67(49.3)$ & $32(30.5)$ & $15(14.3)$ & $7(6.7)$ & $54(51.4)$ & 0.74 \\
\hline Others $N(\%)$ & $12(8.8)$ & $14(10.3)$ & $13(9.5)$ & 39 (28.7) & $19(18.1)$ & $11(10.5)$ & $11(10.5)$ & $42(40.0)$ & 0.07 \\
\hline
\end{tabular}

Data are number of patients (\% of total). Toxicity effects and grades was assessed by Bearman's criteria [40]. Other organs include bladder central nervous system, cardiac and lungs toxicity. Statistical analysis performed by Pearson's chi square.

FBM fludarabine, BCNU/carmustine, melphalan, FTM fludarabine, thiotepa, melphalan.

HCT, patients treated with FTM had a higher frequency of respiratory insufficiency, cardiac/hemodynamic, and septic complications, including admission to the intensive care unit (Supplementary Table 8).

\section{Discussion}

Allo-HCT protocols are in continuous evolution and constantly evaluated for higher efficacy, lower toxicity, and better outcomes [53]. The optimal conditioning regimen for patients with several co-morbidities and/or organ toxicity prior allo-HCT is focus of intense research. The optimization of reduced-intensity/toxicity conditioning protocols might allow allo-HCT to older patients, those with reduced performance status and/or impaired organ function due to prior therapies or disease.

Therefore, we modified our previously described FBM protocol $[19,20]$, which is very effective in patients with high risk of relapse [21], in order to reduce toxicity and improve outcome by replacing BCNU by thiotepa (FTM). Both protocols are based on the very effective fludarabine/ melphalan backbone, for which very low relapse incidence rates in myeloid malignancies are reported [54]. Notably, conditioning with fludarabine, thiotepa, and melphalan have been previously reported by other groups [28-30]. 
Interestingly, no statistically significant differences were observed on adjusted overall survival rates between FBMand FTM-treated patients, despite higher HCT-CI score, more previous chemotherapy treatments and impaired lung function in FTM-treated patients. In the multivariate analysis as well as in subgroup analyses, we could not demonstrate significant differences in OS and NRM between FTM and FBM conditioning. These data suggest that the BCNU can be safely substituted by thiotepa, while maintaining anti-neoplastic effect.

In our study, patients with a HCT-CI score $\geq 4$ have an OS at 24 months of $50 \%$ with both protocols and the cumulative incidence of NRM of $32 \%$ with FBM and $42 \%$ with FTM protocol (Supplementary Fig. 4). Similar data with NRM around $40 \%$ were observed in patients with high HCT-CI score in several studies [31, 55]. Other reducedintensity conditioning protocols using only one alkylating agent as FluBu2 or FluMel show lower NRM rates (about $5 \%$ ) but higher relapse incidence (about $40 \%$ ) at 2 years $[56,57]$. Hence, patients older than 65 years old showed a NRM of $28 \%$ and relapse incidence of $21 \%$ at 24 months with the FBM protocol (Supplementary Fig. 5). Of note, a significant difference for NRM was found in this group of age between both protocols without adjusting for HCT-CI score and other covariates associated with mortality. These data suggest there is still a clinical need for improving conditioning protocols for patients with comorbidities and in advanced age in order to reduce NRM.

Changes in lung function were investigated in patients treated with FBM and FTM. Significant differences between 1 year after allo-HCT and before allo-HCT were found in VCmax, FEV1, FVC and MEF50, suggesting increased lung toxicity in FBM-treated patients. Unexpectedly, lung function parameters as TLC, MEF25, FEV1/ FVC, and DLCOcSB declined similarly in both FBM- and FTM-treated patients surviving at least one year (Fig. 3, Supplementary Table 7). We hypothesize that thiotepa as well as BCNU may cause comparable lung function deterioration at the doses used in our conditioning protocol in some, but not all, lung function parameters.

Both conditioning protocols showed a similar toxicity profile. However, FTM-treated patients suffered more frequently mild to moderate mucositis. Thiotepa has been associated with mucositis in several studies prior auto-HCT [58] and allo-HCT [12, 28-30]. Eder et al. [12] reported a frequency of $46.8 \%$ in 323 ALL patients receiving thiotepa as conditioning. In our cohort, $22.1 \%$ of patients conditioned with FBM received methotrexate but $1.4 \%$ of FTMtreated patients received methotrexate, suggesting that mucositis is not due to concomitant substances given as GvHD prophylaxis. Patients treated with FTM were younger (see Table 1) and patients $<55$ years received 140 $\mathrm{mg} / \mathrm{m}^{2}(16.9 \%$ in FBM and $38.1 \%$ in FTM) while patients $\geq 55$ years old received $110 \mathrm{mg} / \mathrm{m}^{2}$ per conditioning protocols. We hypothesize that higher doses of melphalan in the FTM-cohort might have contributed to the higher frequency of mucositis.

Unexpectedly, we found less HHV6 infections in patients treated with FTM than with FBM. Further subgroup analysis showed that 9 out 20 patients treated with alemtuzumab as GvHD prophylaxis developed HHV6 infections and 19 out of 20 FBM-treated patients received alemtuzumab as GvHD prophylaxis suggesting an association of HHV6 infections with alemtuzumab as confounding variable. Similarly, we found more patients with mixed chimerism at $d+30$ and $d+100$ in FBM-treated compared to FTM-treated patients (Supplementary Table 9), caused by deeper immunosuppression due to alemtuzumab and higher frequency of myeloid disease in the FBM cohort.

This study has several limitations. Significant differences found in univariate analysis on outcome variables between conditioning protocols are probably due to imbalanced clinical characteristics of the patients as age, hematologic malignancies, prior therapies before alloHCT, differences in follow-up and GvHD prophylaxis. In the multivariate analysis and subgroup analysis of patients with clinical features associated with worse prognosis, we did not detect any difference between both conditioning protocols on outcome variables. Second, we performed a retrospective analysis of the patients transplanted at our institution during a specific period of time. There was no randomization of the patients to the conditioning protocols and the selection of the reduced toxicity/intensity protocol was done by choice of the attending physicians depending on disease, prior therapies, and lung function. Therefore, these data should be interpreted with caution. Randomized controlled clinical trials should elucidate which reduced-intensity conditioning protocol is the most effective and the less toxic for specific patient subgroups in the near future.

In conclusion, with FBM and FTM, we have conditioning protocols incorporating two alkylating agents plus fludarabine with an adequate antineoplastic efficacy and suitable for patients with impaired lung function prior alloHCT, several comorbidities, and reduced performance status prior allo-HCT.

Acknowledgements We thank Irmgard Matt for managing the data bank of transplanted patients, the medical technicians in the Stem Cell Transplantation Unit and Pulmonary Function Test Unit of the University Medical Center Freiburg, and all the patients and staff on the Löhr ward. We thank also Elisabeth Lenartz, Stefanie Knebel, and Petra Isele-Hiß for transplantation coordination.

Funding This project was supported by a grant of the German Federal Ministry of Education and Research (BMBF 01 EO 0803). JD-A, MW, and GI received support from Else Kröner-Fresenius-Stiftung (Nr. 2018-A56). JD-A received research support from German Research 
Foundation (Deutsche Forschungsgemeinschaft, ref. DU 1287/3-1), from the Forschungskommission of the University of Freiburg Medical School (DUQ1106/16) and Berta Ottenstein-Programm for Advanced Clinician Scientists, Faculty of Medicine, University of Freiburg.

Author contributions Study concept and design, JD-A and JF. Acquisition of data, JD-A, RZ, RW, HB, JF, TK, JM-Q, RM. Analysis and interpretation of data JD-A, GI, MW, MY, and JF. Critical revision of the manuscript for important intellectual content, JD-A, GI, MW, RZ, RW, MY, T K, JM-Q, RM, and JF. Statistical analysis, JD-A and GI.

\section{Compliance with ethical standards}

Conflict of interest The authors declare no conflicting financial interest with the submission of this article. JF received research support and speakers honoraria from Medac, Neovii and Riemser. JD-A received speakers honoraria from Roche and travel support from Gilead and Sobi. RZ received speakers honoraria from Incyte, Novartis, Roche, and Mallinckroth.

Publisher's note Springer Nature remains neutral with regard to jurisdictional claims in published maps and institutional affiliations.

\section{References}

1. Storb R, Sandmaier BM. Nonmyeloablative allogeneic hematopoietic cell transplantation. Haematologica. 2016;101:521-30.

2. Gyurkocza B, Sandmaier BM. Conditioning regimens for hematopoietic cell transplantation: one size does not fit all. Blood. 2014;124:344-53.

3. Jagannath S, Dicke KA, Armitage JO, Cabanillas FF, Horwitz LJ, Vellekoop L, et al. High-dose cyclophosphamide, carmustine, and etoposide and autologous bone marrow transplantation for relapsed Hodgkin's disease. Ann Intern Med. 1986;104:163-8.

4. Armitage JO, Jagannath S, Spitzer G, Bierman P, Kessinger A, Kumar $\mathrm{P}$, et al. High dose therapy and autologous marrow transplantation as salvage treatment for patients with diffuse large cell lymphoma. Eur J Cancer Clin Oncol. 1986;22:871-7.

5. Sivaraj D, Bacon W, Long GD, Rizzieri DA, Horwitz ME, Sullivan $\mathrm{KM}$, et al. High-dose BCNU/Melphalan conditioning regimen before autologous stem cell transplantation in newly diagnosed multiple myeloma. Bone Marrow Transpl. 2018;53:34-8.

6. Brandes AA, Tosoni A, Amistà P, Nicolardi L, Grosso D, Berti F, et al. How effective is BCNU in recurrent glioblastoma in the modern era? A phase II trial. Neurology. 2004;63:1281-4.

7. Weinkam RJ, Lin HS. Chloroethylnitrosourea cancer chemotherapeutic agents. Adv Pharm Chemother. 1982;19:1-33.

8. Alessandrino EP, Bernasconi P, Colombo A, Caldera D, Martinelli G, Vitulo P, et al. Pulmonary toxicity following carmustinebased preparative regimens and autologous peripheral blood progenitor cell transplantation in hematological malignancies. Bone Marrow Transpl. 2000;25:309-13.

9. Aronin PA, Mahaley MS Jr, Rudnick SA, Dudka L, Donohue JF, Selker RG, et al. Prediction of BCNU pulmonary toxicity in patients with malignant gliomas: an assessment of risk factors. N. Engl J Med. 1980;303:183-8.

10. Emanuel NM, Konovalova NP, Djachkovskaya RF. Toxicity, antitumor activity, and pharmacokinetics of spin-labeled thiotepa analogs. Cancer Treat Rep. 1976;60:1605-9.

11. Heideman RL, Cole DE, Balis F, Sato J, Reaman GH, Packer RJ, et al. Phase I and pharmacokinetic evaluation of thiotepa in the cerebrospinal fluid and plasma of pediatric patients: evidence for dose-dependent plasma clearance of thiotepa. Cancer Res. 1989;49:736-41.

12. Eder S, Beohou E, Labopin M, Sanz J, Finke J, Arcese W, et al. Thiotepa-based conditioning for allogeneic stem cell transplantation in acute lymphoblastic leukemia-A survey from the Acute Leukemia Working Party of the European Society for Blood and Marrow Transplantation. Am J Hematol. 2017;92:18-22.

13. Eder S, Canaani J, Beohou E, Labopin M, Sanz J, Arcese W, et al. Thiotepa-based conditioning versus total body irradiation as myeloablative conditioning prior to allogeneic stem cell transplantation for acute lymphoblastic leukemia: a matched-pair analysis from the Acute Leukemia Working Party of the European Society for Blood and Marrow Transplantation. Am J Hematol. 2017;92:997-1003.

14. Eder S, Labopin M, Finke J, Bunjes D, Olivieri A, Santarone S, et al. Safety and efficacy of thiotepa-based conditioning for allogeneic transplantation in AML: a survey from the ALWP of the EBMT. Bone Marrow Transpl. 2017;52:238-44.

15. Illerhaus G, Marks R, Ihorst G, Guttenberger R, Ostertag C, Derigs G, et al. High-dose chemotherapy with autologous stemcell transplantation and hyperfractionated radiotherapy as first-line treatment of primary CNS lymphoma. J Clin Oncol. 2006;24:3865-70.

16. Schorb E, Kasenda B, Atta J, Kaun S, Morgner A, Hess G, et al. Prognosis of patients with primary central nervous system lymphoma after high-dose chemotherapy followed by autologous stem cell transplantation. Haematologica. 2013;98:765-70.

17. Raiola AM, Van Lint MT, Lamparelli T, Gualandi F, Mordini N, Berisso G, et al. Reduced intensity thiotepa-cyclophosphamide conditioning for allogeneic haemopoietic stem cell transplants (HSCT) in patients up to 60 years of age. $\mathrm{Br} \mathrm{J}$ Haematol. 2000;109:716-21.

18. Lamparelli T, van Lint MT, Gualandi F, Raiola AM, Barbanti M, Sacchi N, et al. Alternative donor transplants for patients with advanced hematologic malignancies, conditioned with thiotepa, cyclophosphamide and antithymocyte globulin. Bone Marrow Transpl. 2000;26:1305-11.

19. Bertz H, Potthoff K, Finke J. Allogeneic stem-cell transplantation from related and unrelated donors in older patients with myeloid leukemia. J Clin Oncol. 2003;21:1480-4.

20. Marks R, Potthoff K, Hahn J, Ihorst G, Bertz H, Spyridonidis A, et al. Reduced-toxicity conditioning with fludarabine, BCNU, and melphalan in allogeneic hematopoietic cell transplantation: particular activity against advanced hematologic malignancies. Blood. 2008;112:415-25.

21. Bertz H, Lübbert M, Ohneberg K, Zeiser R, Wäsch R, Marks R, et al. Allogeneic hematopoietic cell transplantation with double alkylating agents containing reduced-intensity conditioning for patients $\geqslant 60$ years with advanced AML/MDS. Leukemia. 2016;30:2426-9.

22. Deschler B, Binek K, Ihorst G, Marks R, Wäsch R, Bertz H, et al. Prognostic factor and quality of life analysis in 160 patients aged $>$ or $=60$ years with hematologic neoplasias treated with allogeneic hematopoietic cell transplantation. Biol Blood Marrow Transpl. 2010;16:967-75.

23. Deschler B, Ihorst G, Schnitzler S, Bertz H, Finke J. Geriatric assessment and quality of life in older patients considered for allogeneic hematopoietic cell transplantation: a prospective risk factor and serial assessment analysis. Bone Marrow Transpl. 2018;53:565-75.

24. Duque-Afonso J, Ihorst G, Wäsch R, Bertz H, Müller-Quernheim $\mathrm{J}$, Finke J, et al. Identification of risk factors for bronchiolitis obliterans syndrome after reduced toxicity conditioning before hematopoietic cell transplantation. Bone Marrow Transpl. 2013;48:1098-103. 
25. Duque-Afonso J, Ihorst G, Waterhouse M, Zeiser R, Wäsch R, Bertz $\mathrm{H}$, et al. Impact of lung function on bronchiolitis obliterans syndrome and outcome after allogeneic hematopoietic cell transplantation with reduced-intensity conditioning. Biol Blood Marrow Transpl. 2018;24:2277-84.

26. Sellner L, Boumendil A, Finel H, Choquet S, de Rosa G, Falzetti F, et al. EBMT Lymphoma Working Party. Thiotepa-based highdose therapy for autologous stem cell transplantation in lymphoma: a retrospective study from the EBMT. Bone Marrow Transpl. 2016;51:212-8.

27. Joffe E, Rosenberg D, Rozovski U, Perry C, Kirgner I, Trestman $\mathrm{S}$, et al. Replacing carmustine by thiotepa and cyclophosphamide for autologous stem cell transplantation in Hodgkin's and nonHodgkin's B-cell lymphoma. Bone Marrow Transpl. 2018;53: 29-33.

28. Ciurea SO, Saliba R, Rondon G, Pesoa S, Cano P, FernandezVina $\mathrm{M}$, et al. Reduced-intensity conditioning using fludarabine, melphalan and thiotepa for adult patients undergoing haploidentical SCT. Bone Marrow Transpl. 2010;45:429-36.

29. Mehta RS, Di Stasi A, Andersson BS, Nieto Y, Jones R, de Lima $\mathrm{M}$, et al. The development of a myeloablative, reduced-toxicity, conditioning regimen for cord blood transplantation. Clin Lymphoma Myeloma Leuk. 2014;14:e1-5.

30. Bhatt ST, Schulz G, Hente M, Slater A, Murray L, Shenoy S, et al. A single-center experience using alemtuzumab, fludarabine, melphalan, and thiotepa as conditioning for transplantation in pediatric patients with chronic granulomatous disease. Pediatr Blood Cancer. 2020;67:e28030.

31. Sorror ML, Maris MB, Storb R, Baron F, Sandmaier BM, Maloney DG, et al. Hematopoietic cell transplantation (HCT)-specific comorbidity index: a new tool for risk assessment before allogeneic HCT. Blood. 2005;106:2912-9.

32. Gratwohl A. The EBMT risk score. Bone Marrow Transpl. 2012;47:749-56.

33. Copelan E, Casper JT, Carter SL, van Burik JA, Hurd D, Mendizabal AM, et al. A scheme for defining cause of death and its application in the $\mathrm{T}$ cell depletion trial. Biol Blood Marrow Transpl. 2007;13:1469-76.

34. Finke J, Bethge WA, Schmoor C, Ottinger HD, Stelljes M, Zander AR, et al. Standard graft-versus-host disease prophylaxis with or without anti-T-cell globulin in haematopoietic cell transplantation from matched unrelated donors: a randomised, open-label, multicentre phase 3 trial. Lancet Oncol. 2009;10:855-64.

35. Finke J, Schmoor C, Bethge WA, Ottinger H, Stelljes M, Volin L, et al. Long-term outcomes after standard graft-versus-host disease prophylaxis with or without anti-human-T-lymphocyte immunoglobulin in haemopoietic cell transplantation from matched unrelated donors: final results of a randomised controlled trial. Lancet Haematol. 2017;4:e293-e301.

36. Bertz H, Spyridonidis A, Wäsch R, Grüllich C, Egger M, Finke J. A novel GVHD-prophylaxis with low-dose alemtuzumab in allogeneic sibling or unrelated donor hematopoetic cell transplantation: the feasibility of deescalation. Biol Blood Marrow Transpl. 2009; 15:1563-70.

37. Zeiser R, Blazar BR. Acute Graft-versus-host disease - biologic process, prevention, and therapy. N. Engl J Med. 2017;377:2167-79.

38. Zeiser R, Blazar BR. Pathophysiology of chronic graft-versus-host disease and therapeutic targets. N. Engl J Med. 2017;377:2565-79.

39. Glucksberg H, Storb R, Fefer A, Buckner CD, Neiman PE, Clift RA, et al. Clinical manifestations of graft-versus-host disease in human recipients of marrow from HL-A-matched sibling donors. Transplantation. 1974;18:295-304.
40. Bearman SI, Appelbaum FR, Buckner CD, Petersen FB, Fisher $\mathrm{LD}$, Clift RA, et al. Regimen-related toxicity in patients undergoing bone marrow transplantation. J Clin Oncol. 1988;6: 1562-8.

41. Duque-Afonso J, Waterhouse M, Pfeifer D, Follo M, Duyster J, Bertz $\mathrm{H}$, et al. Cell-free DNA characteristics and chimerism analysis in patients after allogeneic cell transplantation. Clin Biochem. 2018;52:137-41.

42. Behringer D, Bertz H, Schmoor C, Berger C, Dwenger A, Finke J. Quantitative lymphocyte subset reconstitution after allogeneic hematopoietic transplantation from matched related donors with CD34+ selected PBPC grafts unselected PBPC grafts or BM grafts. Bone Marrow Transpl. 1999;24:295-302.

43. Calvelli T, Denny TN, Paxton H, Gelman R, Kagan J. Guideline for flow cytometric immunophenotyping: a report from the National Institute of Allergy and Immunologic Diseases, Division of AIDS. Cytometry. 1993;14:702-14.

44. American Thoracic Society. Single-breath carbon monoxide diffusing capacity (transfer factor). Recommendations for a standard technique-1995 update. Am J Respir Crit Care Med. 1995; 152:2185-98.

45. Culver BH, Graham BL, Coates AL, Wanger J, Berry CE, Clarke PK, et al. Recommendations for a Standardized Pulmonary Function Report. An Official American Thoracic Society Technical Statement. Am J Respir Crit Care Med. 2017;196:1463-72.

46. Zaiss AW, Matthys H. A multi user system for whole body plethysmographic measurements and interpretation. Lung. 1990;168:1185-92.

47. Fine J, Gray. R. A proportional hazards model for the subdistribution of a competing 25 risk. J Am Stat Assoc. 1999;94:496-509.

48. Dignam JJ, Kocherginsky MN. Choice and interpretation of statistical tests used when competing risks are present. J Clin Oncol. 2008;26:4027-34.

49. Yates S. Contingency tables involving small numbers and the $\chi^{2}$ test. Suppl J R Stat Soc. 1934;1(No. 2):217-35. pp

50. Down JD, Westerhof GR, Boudewijn A, Setroikromo R, Ploemacher RE. Thiotepa improves allogeneic bone marrow engraftment without enhancing stem cell depletion in irradiated mice. Bone Marrow Transpl. 1998;21:327-30.

51. El-Cheikh J, Labopin M, Al-Chami F, Bazarbachi A, Angelucci E, Santarone S, et al. Effect of the Thiotepa Dose in the TBF conditioning regimen in patients undergoing allogeneic stem cell transplantation for acute myeloid leukemia in complete remission: a report from the EBMT acute leukemia working party. Clin Lymphoma Myeloma Leuk. 2020;20:296-304.

52. Kafaja S, Clements PJ, Wilhalme H, Tseng CH, Furst DE, Kim $\mathrm{GH}$, et al. Reliability and minimal clinically important differences of forced vital capacity: Results from the Scleroderma Lung Studies (SLS-I and SLS-II). Am J Respir Crit Care Med. 2018;197:644-52.

53. Giralt S, Ballen K, Rizzo D, Bacigalupo A, Horowitz M, Pasquini $\mathrm{M}$, et al. Reduced intensity conditioning regimen workshop: defining the dose spectrum. Report of a workshop convened by the center for international blood and marrow transplant research. Biol Blood Marrow Transpl. 2009;15:367-9.

54. Eapen M, Brazauskas R, Hemmer M, Perez WS, Steinert P, Horowitz MM, et al. Hematopoietic cell transplant for acute myeloid leukemia and myelodysplastic syndrome: conditioning regimen intensity. Blood Adv. 2018;2:2095-103.

55. Muffly L, Pasquini MC, Martens M, Brazauskas R, Zhu X, Adekola K, et al. Increasing use of allogeneic hematopoietic cell transplantation in patients aged 70 years and older in the United States. Blood. 2017;130:1156-64. 
56. Scott BL, Pasquini MC, Logan BR, Wu J, Devine SM, Porter $\mathrm{DL}$, et al. Myeloablative versus reduced-intensity hematopoietic cell transplantation for acute myeloid leukemia and myelodysplastic syndromes. J Clin Oncol. 2017;35: 1154-61.

57. Scott BL. Long-term follow up of BMT CTN 0901, a randomized phase III trial comparing myeloablative (MAC) to reduced intensity conditioning (RIC) prior to hematopoietic cell transplantation (HCT) for acute myeloid leukemia (AML) or myelodysplasia (MDS) (MAvRIC Trial). Biol Blood Marrow Transpl. 2020. https://doi.org/10.1016/j.bbmt.2019.12.075.

58. Ferreri AJM, Cwynarski K, Pulczynski E, Fox CP, Schorb E, La Rosée P, et al. Whole-brain radiotherapy or autologous stem-cell transplantation as consolidation strategies after high-dose methotrexate-based chemoimmunotherapy in patients with primary CNS lymphoma: results of the second randomisation of the International Extranodal Lymphoma Study Group-32 phase 2 trial. Lancet Haematol. 2017;4:e510-e523. 